\title{
Supercritical carbon dioxide paper artifacts cleaning
}

\author{
Shi-wei Su \\ North China Electric Power University, Baoding 071003, China \\ 2446567415@qq.com
}

\begin{abstract}
This paper mainly introduces the current paper artifacts cleaning technology, pointing out the drawbacks and damage of traditional craft. Introduction of supercritical carbon dioxide $\left(\mathrm{SCCO}_{2}\right)$ and its application. The use of $\mathrm{SCCO}_{2}$ between the gas and the special nature of the liquid, $\mathrm{SCCO}_{2}$ for the cleaning of paper artifacts, and in this supercritical fluid painting and calligraphy on the basis of cleaning, combined with the drawbacks of the existing painting and calligraphy, Clarify the advantages of $\mathrm{SCCO}_{2}$ for painting and calligraphy cleaning.
\end{abstract}

Keywords: Supercritical carbon dioxide, paper artifacts, cleaning.

\section{Introduction}

At present, domestic and foreign use of ancient cleaning methods are mainly solution or latex attached to a layer of polymer, such as acrylic polymer, starch, plant gum, chitosan and so on. This traditional cleaning method of cleaning fluid can not be separated from the water, although the paper can be whiteness, $\mathrm{pH}$ value has increased, but the water will seriously damage the tensile strength and degree of polymerization, for the protection of calligraphy and painting is very unfavorable. And will be dirty environment, easy to hurt and heart (on the damage to a serious impact on the heart more) and secondary pollution and other issues. The traditional calligraphy and painting machine is a solvent for water and has not yet designed a painting and washing machine suitable for $\mathrm{SCCO}_{2}$ as solute.

As the supercritical carbon dioxide cleaning technology environmental protection, high efficiency and other advantages, the current $\mathrm{SCCO}_{2}$ cleaning technology has been applied in the following areas: printed circuit boards, silicon chips and other electronic devices; cleaning precision instruments; cleaning optical lenses; medical equipment industry; Of the residual pesticides; treatment of nuclear materials ${ }^{[1]}$. But there is not a specific program to use $\mathrm{SCCO}_{2}$ for painting and calligraphy. This paper provides a concrete solution for the cleaning of paper artifacts by $\mathrm{SCCO}_{2}$, and discusses the feasibility and advantages of the system.

\section{Traditional cleaning technology and defects}

The current cleaning water can be warm water can also use the "room temperature water", for the more stubborn stains, in ancient times there is the use of Gleditsia water rinse the screen practice, which can play a certain picture bleaching effect; in modern times, you can also use chemistry Pharmacy for painting and calligraphy for cleaning, bleaching or deacidification treatment ${ }^{[2]}$. Chemical decontamination, you can remove the traditional washing method is difficult to 
remove the oil traces, waxy spot, mildew, rust and other stubborn stains. For the removal of stains, wax spots and other stains, the principle is the use of agents on the stains of the dissolution of the pollutants after the transfer of pollutants transferred to the decontamination paper to achieve the purpose of decontamination. And for the removal of plaques and other pigments, is the use of pharmaceutical bleaching, the screen stains fade, bleach can also be used to bleach the yellow paper, bleach an oxidized bleach and reducing bleach.

Except for aqueous solution, there are organic solvents rinse. Organic solvents to volatile solvents and halogen-containing chlorofluorocarbon solvent-based, the world every year to use millions of tons, the destruction of the ozone layer, the atmospheric environment pollution is very serious. The Montreal Protocol, signed in 1987 by the world, has set out the ban on such solvents ${ }^{[3]}$. Aqueous solution cleaning requires a complex surfactant formulation, which can form secondary pollution for a long drying time, and must also increase equipment and handling costs.

In the traditional cleaning method, because of the surface tension and viscosity of the cleaning medium, it is very difficult to clean thoroughly when cleaning the very small pores. The cleaning process requires the use of chemical reagents, which have a significant impact on the environment and the health of the operators. Traditional washing for paper artifacts will have a very serious damage, is not conducive to long-term preservation of cultural relics, and the cleaning process will consume a lot of water, resulting in waste of water resources.

\section{$3 \mathrm{SCCO}_{2}$ Analysis of the Feasibility of Washing paper artifacts}

Temperature and pressure are above the critical point of the liquid called supercritical fluid. The temperature and pressure of the critical point of the supercritical state of different substances are not the same. Supercritical fluids have the characteristics of low viscosity, high diffusion, high density and low surface tension, which make them have better dissolving ability and strong flowability. The critical temperature and pressure of carbon dioxide are $31.1^{\circ} \mathrm{C}$ and $7.38 \mathrm{MPa}$, respectively. When the temperature and pressure of carbon dioxide are more than its critical temperature and pressure, the carbon dioxide enters the supercritical state, where the carbon dioxide has the carbon dioxide and liquid properties. Carbon dioxide supercritical state and its gas, liquid properties of comparison, has the following characteristics:

1)the coefficient is more than 10 times the liquid, with a large diffusion capacity, the material has a very good permeability;

2)small viscosity, good transfer, fast moving speed;

3)viscosity are important parameters to measure the mass transfer capacity of supercritical fluid. Therefore, the characteristics of supercritical carbon dioxide determine its good mass transfer ability ${ }^{[4]}$.

Supercritical fluid extraction technology for the use of natural oil has a large number of deacidification, the ancient carrier for the paper, it has similar properties with other natural substances, the use of supercritical fluid high permeability, penetrate into the ancient books, The paper in the acidic material extracted, while the 
deacidification agent into the whole of the ancient paper gap, so that the paper has a certain amount of deacid residue, delay the production of acidification. So the supercritical extraction technology into the paper deacidification, technically feasible ${ }^{[5]}$.

\section{4 supercritical carbon dioxide cleaning advantages}

$\mathrm{SCCO}_{2}$ as a special cleaning agent, not only has high solubility, high diffusion, low viscosity, low surface tension and other characteristics, but also water-based solutions and organic solvents excellent green alternatives. $\mathrm{SCCO}_{2}$ can be in the paper of microbial metabolites, dust and other effective substances removed. Not only improve the $\mathrm{pH}$ value, the alkaline residue also increased to some extent, making the paper's acid resistance increased. $\mathrm{SCCO}_{2}$ cleaning calligraphy and painting can effectively inhibit the spread of ink, to protect the original appearance of the estimate. The cleaning process can be cleaned thoroughly without having to open the book binding. The advantages of using supercritical carbon dioxide to clean paper artifacts are:

(1) $\mathrm{SCCO}_{2}$ surface tension is very small, similar to zero, $\mathrm{SCCO}_{2}$ this feature can make up for the lack of traditional cleaning technology, and then break through the bottleneck of traditional cleaning methods, and thus into the cultural relics inside the cleaning.

(2) Can be in the room temperature and carbon dioxide gas surrounded by cleaning, effective cleaning of oil and other pollutants, carbon dioxide is not active gas, in the cleaning process generally does not occur chemical reaction, cleaning parts will not be due to oxidation and corrosion.

(3) $\mathrm{SCCO}_{2}$ is the cleanest cleaning medium, cleaning the whole process to avoid the use of chemical additives, such as chlorofluorocarbons, this chemical can cause ozone holes, to avoid the extraction process of human damage and environmental pollution.

(4) Cleaning and drying process in one, when the $\mathrm{SCCO}_{2}$ containing dissolved material flows through the separation tank, the separation of the pressure drop makes the $\mathrm{SCCO}_{2}$ and the cleaning parts are immediately divided into two phases and quickly separated, not only Improve the efficiency of cleaning and their own less energy consumption, saving economic costs.

(5) Temperature and pressure can be adjusted to adjust the cleaning process parameters. At the critical point, subtle changes in pressure temperature can cause changes in the density of carbon dioxide, making the cultural pollutants solubility changes, through the control of pressure or temperature to achieve cleaning objectives. If the temperature is fixed, reduce the pressure can be separated from the cleaning; the other hand, if the pressure is fixed, change the temperature can also be separated from the cleaning, so cleaning process is short, time-consuming.

(6) $\mathrm{SCCO}_{2}$ cleaning device technology safe and reliable, to protect the environment. Carbon dioxide Chemical nature Inactive, tasteless, non-toxic, colorless, safe. Clean personnel from highly combustible, corrosive and toxic. In the conservation of water resources at the same time, minus the treatment of waste water pollutants steps, both environmentally friendly, energy saving, but also to minimize the pressure on the 
environment.

(7) Carbon dioxide is cheap, has a wide range of sources, and many are by-products of factory production, which can be prevented from being discharged into the air and reducing the greenhouse effect. Carbon dioxide can be recovered from gas, coal-fired steel plants or power plants and can also be recovered from cement lime furnaces and alcoholic fermentation ${ }^{[6]}$.

\section{Conclusions}

The traditional paper artifact cleaning technology is no longer able to meet the requirements of cultural relics protection. Supercritical carbon dioxide is a kind of special fluid with excellent properties, and it has been widely used in many fields. If the supercritical carbon dioxide for paper artifact cleaning with non-toxic harmless, good environment, recyclable; surface tension is low, strong diffusion, you can clean the nanoparticles; after cleaning without drying, only decompression can Completely remove the carbon dioxide; cleaning temperature is low, no damage to the paper artifacts.

\section{References}

[1] Zhao Peng. Supercritical carbon dioxide extraction and coating experimental device design and key equipment internal flow field simulation [D]. Qingdao University of Science and Technology, 2012.

[2] Zhang Xuanwei, Geng Miao. "For the new Yan" do not forget to "buy insurance" painting and calligraphy and cultural relics repair and deacidification [J]. Art market, 2009,12: 86-87.

[3] Yang Haiou, Yang base. Supercritical CO 2 cleaning machine on the lubricant of the preliminary study [J]. Fine Petrochemical, 2003,05: 32-36.

[4] Huang Luojun, Kang Heng, Cheng Song, Li Yongtao, Xia Yang, Jing Yupeng.Application of supercritical CO_2 cleaning technology in CMOS image sensor [J]. Microelectronics Technology, 2017,04: 273-278 + 290 The

[5] Wang Yanjuan. Supercritical carbon dioxide in the ancient paper deodorization and strengthening of the behavior of [D]. Guangdong University of Technology, 2012.

[6] Ningzhao wide. Ultrasonic assisted supercritical CO 2 parts cleaning device development [D]. Qingdao University of Science and Technology, 2014. 